\title{
Correlation femtoscopy at NICA energies
}

\author{
Konstantin Mikhaylov ${ }^{1,2, *}$, Pavel Batyuk ${ }^{2, * *}$, Olga Kodolova ${ }^{3, * * *}$, Lyudmila Malinina ${ }^{2,3, * * * *}$, \\ Grigory Nigmatkulov ${ }^{4, \dagger}$, and Gleb Romanenko ${ }^{2, \ddagger}$ \\ ${ }^{1}$ NRC Kurchatov Institute - ITEP, Rusian Federation, Moscow, Russia, \\ ${ }^{2}$ Joint Institute for Nuclear Research, Dubna, Russia, \\ ${ }^{3}$ Skobeltsyn Research Institute of Nuclear Physics, Moscow State University, Moscow, Russia, \\ ${ }^{4}$ National Research Nuclear University MEPhI, Moscow, Russia.
}

\begin{abstract}
.
The space-time characteristics of particle production are measured by the correlation femtoscopy with use of quantum statistics (QS) and final state interactions (FSI) effects. Femtoscopy at lower energy was a key of importance at AGS, SPS and in the Beam Energy Scan (BES) physics program at RHIC.

Using femtoscopy observables we investigate possibilities to observe a difference from the first-order phase transition expected, according to some theoretical predictions, at low energies and the cross over one, to be occurred at high energies. The hybrid model vHLLE+UrQM is used as a baseline for this study. The possibilities to use kaon femtoscopy complementary to the usually used pion one are discussed.
\end{abstract}

\section{Introduction}

Femtoscopic correlations allows to reach directly the spatial and temporal scales of the extremely small and short-lived systems born in particle and nuclei collisions. The accuracy that one may achieve is about $1 \mathrm{fm}$.

A set of existing (BM@N) and planed experiments (MPD, SPD) are situated at collider NICA with the beam energy $\sqrt{s_{N N}}=4-11 \mathrm{GeV}$. The high luminosity planed for NICA operation will allow to extend the commonly used pion femtoscopic correlation program also to the heavier particles.

The recent theoretical studies ([1]-[3]) suppose that the nuclear matter may come into the first-order phase transition already at NICA energies. As it is proposed in Ref. [4], the firstorder phase transition leads to a stalling of the expansion speed and increase of the emission duration, $\Delta \tau$ what, in turn, results in increasing both $R_{\text {long }}$ and $R_{\text {out }} / R_{\text {side }}$.

Femtoscopy technique applied to the same-charge pions within BES physics program reveals the maximum in the excitation function of $R_{\text {out }} / R_{\text {side }}$ at around $\sqrt{s_{N N}}=20 \mathrm{GeV}$ [5]. The existence of maximum may be attributed to a possible expected change of nuclear matter

\footnotetext{
*e-mail: Konstantin.Mikhaylov@ cern.ch

**e-mail: Pavel.Batyuk@jinr.ru

***e-mail: Olga.Kodolova@cern.ch

****e-mail: Ludmila.Malinine@ cern.ch

†e-mail: nigmatkulov@gmail.com

†e-mail: Gleb.Romanenko@cern.ch
} 

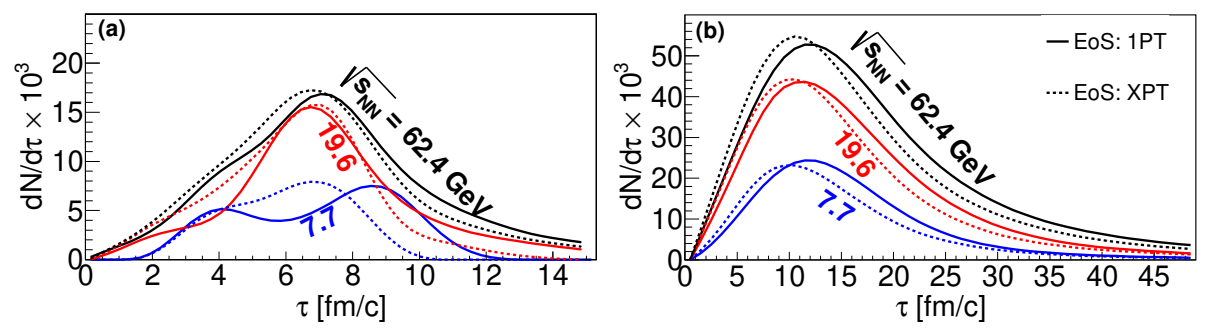

Figure 1. Pion emission times at the particlization surface (a) and the last interactions (b) in the centerof-mass system of colliding gold nuclei at different values of $\sqrt{s_{N N}}$.

equation of state (EoS) and type of phase transition. In the contrary to the high energies, according to theoretical predictions the nuclear matter at the NICA energy range is a baryon dominated. Applying femtoscopy technique at NICA energy we may reveal the phase transition occured in high-baryonic density regime.

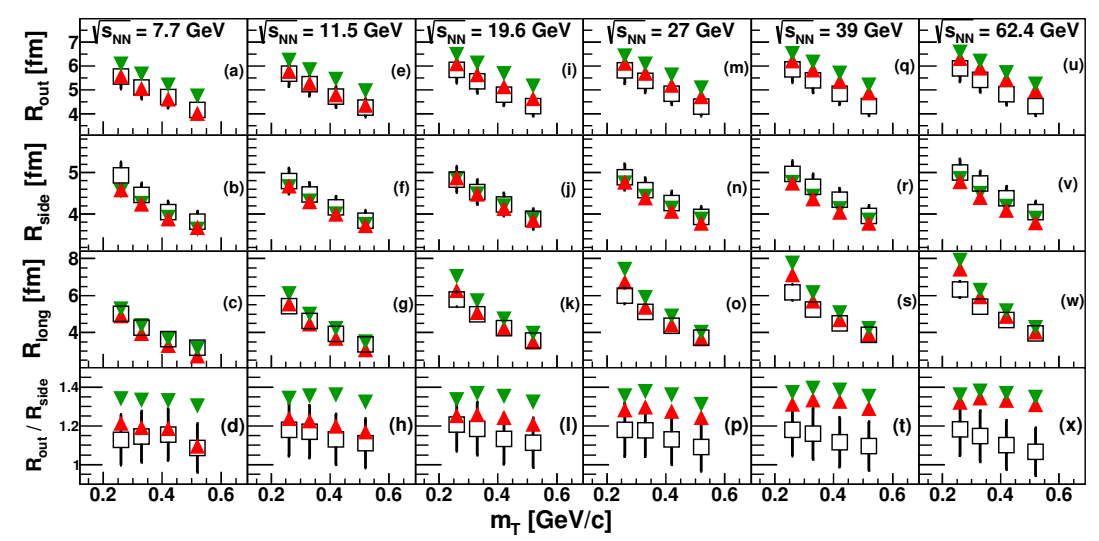

Figure 2. Comparison of the model pion femtoscopy radii with those obtained from the BES program at $\sqrt{s_{N N}}=7.7((\mathrm{a})-(\mathrm{d})), 11.5((\mathrm{e})-(\mathrm{h})), 19.6((\mathrm{i})-(\mathrm{l})), 27((\mathrm{~m})$ - (p)), $39((\mathrm{q})-(\mathrm{t})), 62.4((\mathrm{u})-(\mathrm{x})) \mathrm{GeV}$. Open squares represent experimental data. Green and red triangles correspond to the 1PT EoS and XPT EoS respectively.

Detailed study on the possibility to observe the phase transition and the dense matter characterstics is performed with the hybrid Monte Carlo generator vHLLE+UrQMD model [6]. This model was tuned with existing experimental data to be able to reproduce quantitatively well a set of bulk observables (yields, $p_{T}$ and rapidity spectra). vHLLE+UrQMD model has an option to choose between two EoSs [7, 8] and demonstrates the dependence of the average pion creation time at the particlization surface and the last interactions on collision energy as a function of the EoS (Fig. 1). The calculations involving the first-order phase transition are denoted by 1PT, meanwhile those ones corresponded to the cross over EoS are denoted by XPT. Although the dependence looks apparently weak and the hadronic cascade smears the visible difference the model can be used for studying observables being sensitive to EoS scenario. 


\section{Results and discussion}

Continuing study on extraction of the femtoscopy radii from three-dimensional correlation functions done in Ref. [9] we extracted the femtoscopic radii, $R_{\text {out }, \text { side, long, }}$, the difference, $R_{\text {out }}^{2}-R_{\text {side }}^{2}$, and the ratio, $R_{\text {out }} / R_{\text {side }}$ and compared with existing experimental data on pion femtoscopy obtained from the BES program [5] as a function of collision energy.

The used parametrization of correlation function is a standard one described in [10,11].

Fig. 2 demonstrates that the model reasonably describes the $m_{T}$-dependence of radii for all available energies for both scenarios of the EoS. The radii demonstrate different trends in the out, side and long directions while the $R_{\text {side }}$ is similar for both scenarios. At the same time $R_{\text {out }}$ has a tendency to be larger for about $0.5 \mathrm{fm}$ in the first order phase transition EoS. A longer lifetime of the fluid phase in the first order phase transition EoS leads to bigger values of $R_{\text {long }}$ with respect to cross over EoS.

The ratio of femtoscopic radii extracted from simulated data with the first order phase transition EoS to radii found with cross over EoS are shown in Fig. 3. $R_{\text {side }}$ coincides for both scenarios while $R_{\text {out }}$ and $R_{\text {long }}$ are larger for the first order phase transition EoS and demonstrate a strong $k_{T}$-dependence. That confirms that for the first order phase transition EoS the radial flow developed in the hydro phase of collision is weaker than for the cross over EoS and, therefore, emphasizing a strong role of radial flow effects.

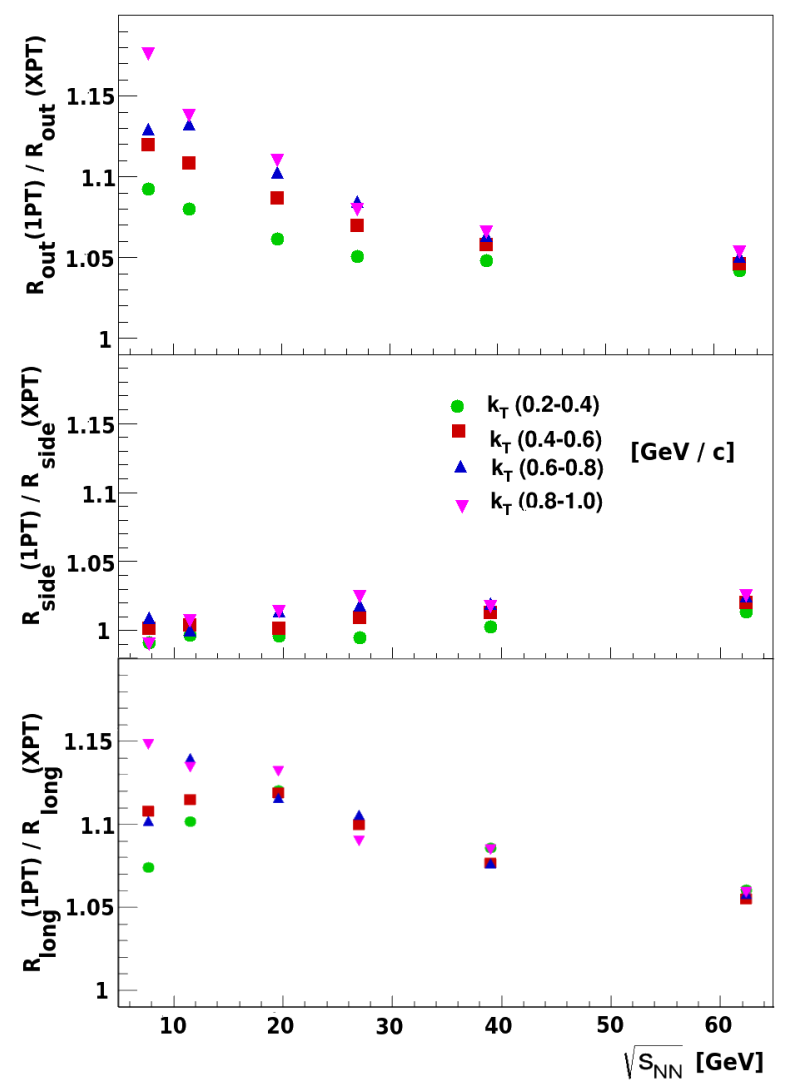

Figure 3. Ratio of femtoscopic radii calculated for the two available scenarios of EoS. 

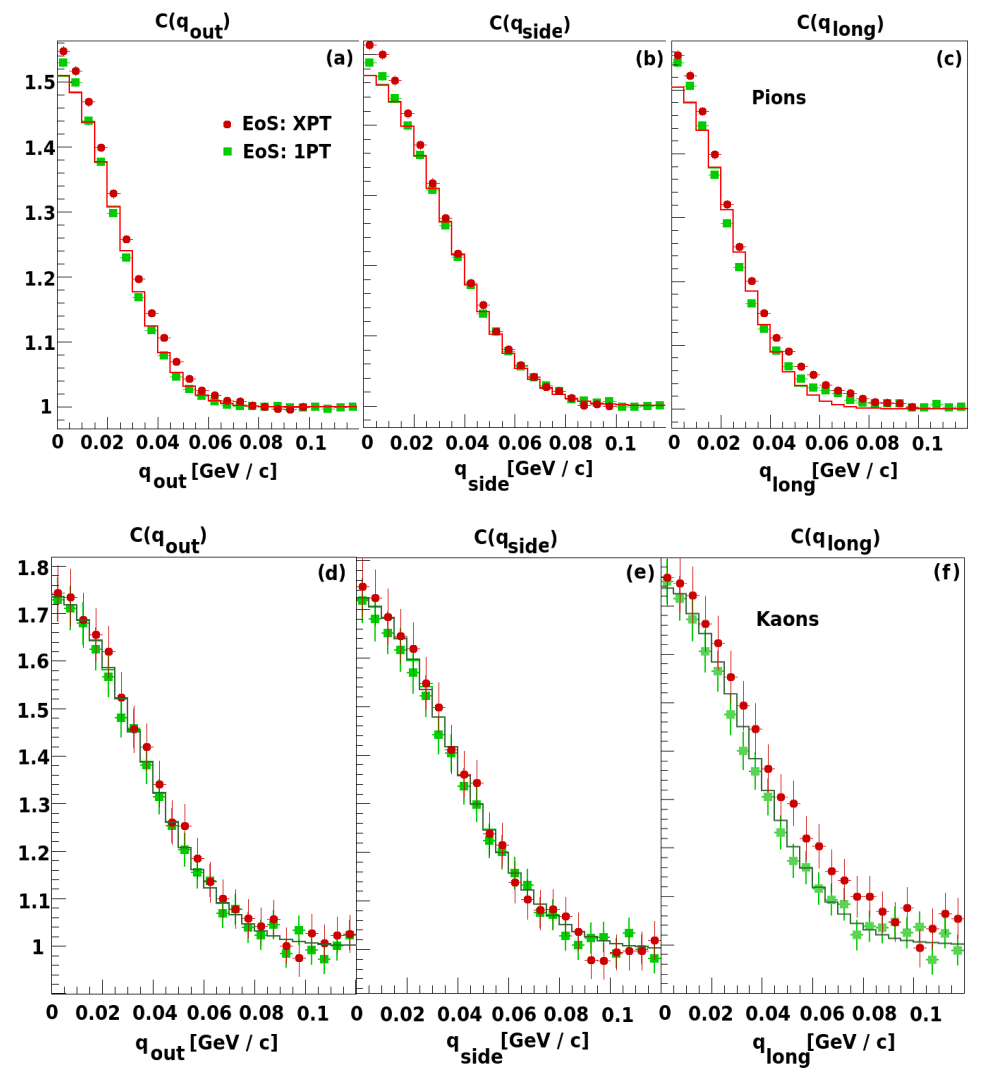

Figure 4. Projections of three-dimensional correlation functions for pions ((a) - (c)) and kaons ((d) - (f)) on out, side and long directions. The calculations made at $\sqrt{s_{N N}}=11.5 \mathrm{GeV}$.

We extend our study introducing the femtoscopy technique to kaons. One of the advantages of this extension is the much less contribution from the resonances decay products in the same-sign kaons sample.

Projections of kaon three-dimensional correlation functions on out, side and long directions are shown in Fig. 4 ((d) - (f)) in comparison with pion ones (upper row of Fig. 4).

We approximate Gaussian radii with the parametrization of correlation function proposed in Ref. [9].

The kaon projections on all directions are more Gaussian then pion ones. Cross over EoS scenario leads to wider projections with respect to those resulted from first order phase transition EoS. This effect is more significant for kaons.

Figure 5 shows the $m_{T}$-dependence of the extracted kaon and pion radii for two energies in the NICA energy range and both possible scenarios of EoS. Data for pions got within BES program are presented on the same plots. The general behaviour of the kaon and pion radii from the model are very similar. For both cases the radii and the $R_{\text {out }} / R_{\text {side }}$ ratio corresponding to the first order phase transition EoS are larger than those ones for the cross over EoS. At the same time the kaon $R_{\text {out }}$ has a tendency to diverge for the two types of EoS with increase of $m_{T}$. It may be attributed to the weak radial flow developed in the hydro phase of collision, but it is just an assumption since it has not been investigated yet in detail and is planned to be done in the future. 

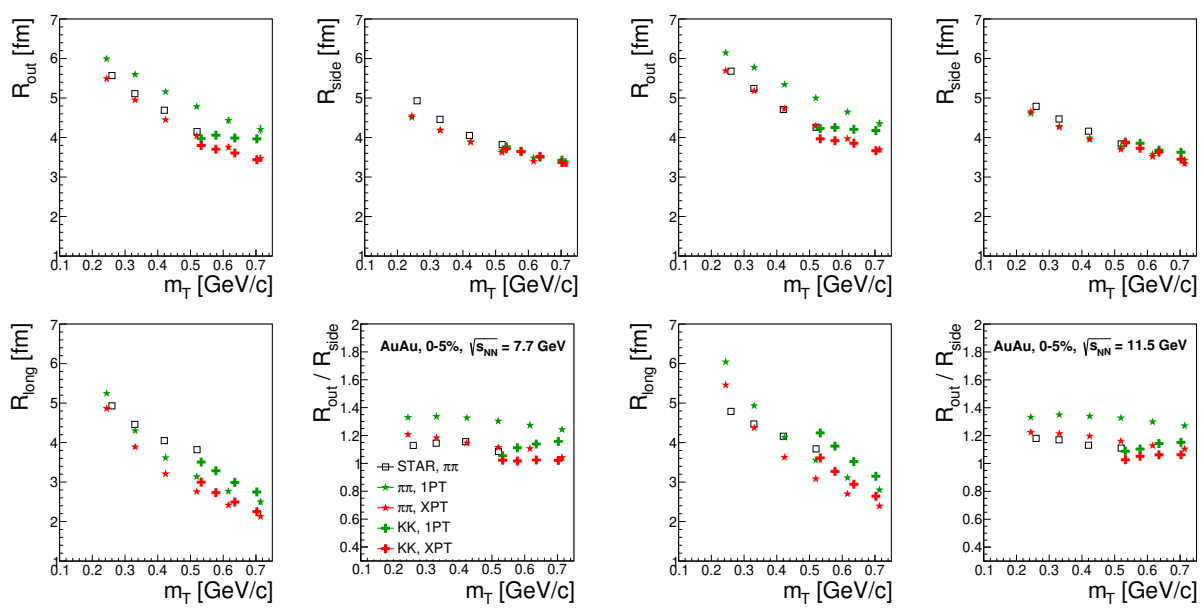

Figure 5. Comparison of the model pion and kaon femtoscopy radii for two energies $\sqrt{s_{N N}}=7.7,11.5$ $\mathrm{GeV}$ close to the NICA energy range with pion data got from the BES program.

\section{Acknowledgments}

This work has been supported by the grant of Russian Foundation for Basic Research (1802-40044) and also partially supported by the National Research Nuclear University MEPhI in the framework of the Russian Academic Excellence Project (contract No. 02.a03.21.0005, 27.08.2013). The authors would like to thank Yu. Sinyukov, Yu. Karpenko and L. Rednický for their great contribution to the work and possibilities to have fruitful discussions aimed at doing the work clearer and better.

\section{References}

[1] J.Berges and K.Rajagopal, Nucl. Phys. B 538 (1999) 215.

[2] M.A.Halasz et al. Phys. Rev. D 58 (1998) 096007.

[3] O.Scavenius, A.Mocsy, I.N.Mishustin and D.H.Rischke, Phys. Rev. C 64 (2001) 045202.

[4] D. H. Rischke and M. Gyulassy, Nucl. Phys. A 608, 479 (1996).

[5] L. Adamczyk et al. [STAR Collaboration], Phys. Rev. C 92, no. 1, 014904 (2015).

[6] I. A. Karpenko, P. Huovinen, H. Petersen and M. Bleicher, Phys. Rev. C 91, no. 6, 064901 (2015).

[7] J. Steinheimer, S. Schramm and H. Stocker, J. Phys. G 38, 035001 (2011).

[8] P. F. Kolb, J. Sollfrank and U. W. Heinz, Phys. Rev. C 62, 054909 (2000).

[9] P. Batyuk, I. Karpenko, R. Lednicky, L. Malinina, K. Mikhaylov, O. Rogachevsky and D. Wielanek, Phys. Rev. C 96, no. 2, 024911 (2017).

[10] S. Pratt, Phys. Rev. D 33, 1314 (1986).

[11] G. Bertsch, M. Gong and M. Tohyama, Phys. Rev. C 37, 1896 (1988).

[12] A. V. Baranov, N. A. Balashov, N. A. Kutovskiy and R. N. Semenov, Phys. Part. Nucl. Lett. 13, no. 5, 672 (2016). 